\title{
Impact of Hemorheology Assessed by the Microchannel Method on Pulsatility Index of the Common Carotid Artery in Patients With Type 2 Diabetes Mellitus
}

\author{
Takashi Hitsumoto
}

\begin{abstract}
Background: Type 2 diabetes mellitus is known to be closely associated with the risk of ischemic stroke. Recent clinical studies have reported that a high pulsatility index (PI) of the cerebral or carotid artery, which is estimated by ultrasonography, also reflects a risk of ischemic stroke. This cross-sectional study aimed to clarify the impact of hemorheology assessed by the microchannel method on the PI of the common carotid artery (CCA) in patients with type 2 diabetes mellitus in terms of the primary prevention of ischemic stroke.
\end{abstract}

Methods: In total, 349 outpatients on treatment for type 2 diabetes mellitus (131 men and 218 women; mean age \pm standard deviation: $65 \pm 11$ years) with no history of cardiovascular events, including ischemic stroke, were enrolled. The whole blood passage time (WBPT) as a marker of hemorheology and the PI of CCA were measured using commercial devices, and their relationships to various clinical parameters were examined.

Results: A significant positive correlation was observed between WBPT and the PI of CCA ( $\mathrm{r}=0.49, \mathrm{P}<0.001)$. Furthermore, multivariate analysis revealed that patients with high WBPT $(\geq 70 \mathrm{~s})$ had significantly higher risk (odds ratio: 5.2; 95\% confidence interval: 2.4 - 9.2; P $<0.001)$ of being detected with a high PI of CCA $(\geq 2)$ than those with low WBPT $(\leq 52.0 \mathrm{~s})$.

Conclusion: The results of this study indicated that WBPT was an important determination factor for the PI of CCA, suggesting that an increase in WBPT can potentially predict the incidence of ischemic stroke in patients with type 2 diabetes mellitus.

Keywords: Hemorheology; Microchannel method; Pulsatility index; Skin autofluorescence; Oxidative stress; Smoking; Type 2 diabetes mellitus

\section{Introduction}

It is well known that type 2 diabetes mellitus is closely associ-

Manuscript accepted for publication April 12, 2017

Hitsumoto Medical Clinic, 2-7-7, Takezakicyou, Shimonoseki City, Yamaguchi 750-0025, Japan. Email: thitsu@jcom.home.ne.jp

doi: https://doi.org/10.14740/jocmr3031w ated with lifestyle and is one of the most important risk factors for ischemic stroke [1]. Furthermore, some clinical studies have reported that patients with type 2 diabetes mellitus and ischemic stroke have poor prognoses [2,3]. Therefore, it is crucial to employ methods for preventing ischemic stroke in patients with type 2 diabetes mellitus at early stages.

Pulsatility index (PI) is a hemodynamic parameter that is determined by Doppler sonography and reflects the degree of vascular resistance. Some clinical studies have reported that the PI of the carotid or cerebral artery is associated not only with arteriosclerosis of cerebral vessels but also incidence of ischemic stroke $[4,5]$. During an ultrasonography examination of the carotid or cerebral artery, the common carotid artery (CCA) can be easily detected in a clinical setting. Furthermore, Nakatou et al have reported that an increase in the PI of CCA reflects a risk of ischemic stroke [5].

The impairment of hemorheology is an important factor for the incidence of cardiovascular events [6, 7]. Recently, a commercial device called microchannel array flow analyzer (MC-FAN), which evaluates hemorheology using microscopic images, has been established and is being used in clinical settings [8]. Using MC-FAN is simple, and it is superior to other methods in terms of the accuracy of channel dimensions and high reproducibility $[8,9]$. Furthermore, some clinical studies have reported a significant relationship between an increase in the whole blood passage time (WBPT), which is measured using MC-FAN, and arterial dysfunction $[10,11]$. Thus, the increase in WBPT possibly reflects an elevation in the PI of the cerebral or carotid artery, which consequently causes ischemic stroke.

To the best of our knowledge, there are no reports on the relationship between WBPT and PI or ischemic stroke. Therefore, this study examined the relationship between WBPT and the PI of CCA in patients with type 2 diabetes mellitus in terms of the primary prevention of ischemic stroke.

\section{Materials and Methods}

\section{Patients}

This cross-sectional study was conducted at the Hitsumoto Medical Clinic in Shimonoseki City from July 2013 to June 2016. The study population comprised 349 outpatients on treatment for type 2 diabetes mellitus who underwent an ultrasonographic examination of carotid arteries. No patient had a 
history of cardiovascular events, such as ischemic stroke, coronary artery disease, peripheral arterial disease, or atrial fibrillation. The patients comprised 131 men and 218 women with a mean age \pm standard deviation (SD) of $65 \pm 11$ years. All participants provided informed consent, and the study protocol conformed to the ethical guidelines of the Declaration of Helsinki. The study was approved by the Local Ethics Committee of the Hitsumoto Medical Clinic.

\section{Evaluation of hemorheology using MC-FAN}

The evaluation of hemorheology was performed by measuring WBPT using MC-FAN HR300 rheometer (MC Healthcare Inc., Tokyo, Japan), as previously reported $[8,12]$. Briefly, the microchannel passage time for $100-\mu \mathrm{L}$ physiological saline as a control was initially measured, followed by that for $100-\mu \mathrm{L}$ heparinized blood samples obtained from study participants. WBPT of the participants was corrected for the passage time of physiological saline. Microchannel formation had a width of 7 $\mu \mathrm{m}$, a length of $30 \mu \mathrm{m}$, and a depth of $4.5 \mu \mathrm{m}$. WBPT measurements were performed within $60 \mathrm{~min}$ of blood sampling. Inter- and intra-assay coefficients of variation for WBPT were $8 \%$ and $5 \%$, respectively.

\section{Ultrasonographic examination of carotid artery}

Ultrasonographic examination of carotid arteries was performed using a high-resolution ultrasonographic scanner with a 9-MHz linear array transducer (HI VISION Avius, Hitachi Medical Corporation, Tokyo, Japan). The PI of CCA was measured as previously reported [5]. Briefly, the pulsed wave Doppler measurements were performed with the sample volume located in the middle CCA region with a maximum Doppler angle of $60^{\circ}$. Using a cine-loop function, the peak systolic velocity (PSV), end diastolic velocity (EDV), and time-averaged velocity (TAV) were calculated by software, and PI was automatically calculated as follows: PI $=((\mathrm{PSV}-\mathrm{EDV}) / \mathrm{TAV})$. The average of PI values of the right and left carotid arteries was calculated and defined as PI. The maximum intima-media thickness of all carotid arteries was defined as max-IMT, as previously reported [13]. In the present study, no patient had total occlusion of CCA or internal carotid artery.

\section{Evaluation of cardiovascular risk factors}

The degree of obesity was estimated using body mass index, which was calculated as weight in kilograms divided by height in meters squared. Current smoking was defined as smoking at least one cigarette per day during the previous 28 days. The right brachial blood pressure was measured twice using a mercury sphygmomanometer with the participants in the sitting position. The average of two readings was used to determine systolic and diastolic blood pressures. Skin autofluorescence (AF), which reflects the accumulation of advanced glycation end products (AGEs), was measured on the volar side of the
Table 1. Baseline Clinical Characteristics

\begin{tabular}{|c|c|}
\hline $\mathrm{n}$ (male/female) & $349(131 / 218)$ \\
\hline Age (years) & $65 \pm 11$ \\
\hline Body mass index & $24.0 \pm 3.8$ \\
\hline Current smoker, n (\%) & $79(23)$ \\
\hline Systolic blood pressure (mm Hg) & $141 \pm 17$ \\
\hline Diastolic blood pressure (mm Hg) & $82 \pm 11$ \\
\hline White blood cell $(/ \mu \mathrm{L})$ & $6,490 \pm 1,370$ \\
\hline Red blood cell $\left(10^{4} / \mu \mathrm{L}\right)$ & $426 \pm 41$ \\
\hline Hematocrit (\%) & $38.4 \pm 4.0$ \\
\hline Platelet $\left(10^{4} / \mu \mathrm{L}\right)$ & $21.9 \pm 6.4$ \\
\hline Fasting blood glucose (mg/dL) & $131 \pm 27$ \\
\hline Immunoreactive insulin $(\mu \mathrm{g} / \mathrm{mL})$ & $7.4 \pm 4.6$ \\
\hline HbA1c (\%) & $7.0 \pm 0.8$ \\
\hline HOMA-IR & $2.5 \pm 1.6$ \\
\hline Skin autofluoresence (AU) & $2.6 \pm 0.5$ \\
\hline Total cholesterol (mg/dL) & $215 \pm 39$ \\
\hline LDL-cholesterol (mg/dL) & $138 \pm 38$ \\
\hline Triglyceride (mg/dL) & $137 \pm 64$ \\
\hline HDL-cholesterol (mg/dL) & $49 \pm 14$ \\
\hline eGFR $\left(\mathrm{mL} / \mathrm{min} / 1.73 \mathrm{~m}^{2}\right)$ & $60 \pm 21$ \\
\hline d-ROMs test (U.Carr) & $333 \pm 98$ \\
\hline PI of CCA & $1.69 \pm 0.63$ \\
\hline Max-IMT & $1.4 \pm 0.4$ \\
\hline WBPT (s) & $62.9 \pm 18.0$ \\
\hline \multicolumn{2}{|l|}{ Medication } \\
\hline Sulfonylurea, n (\%) & $188(54)$ \\
\hline DPP-4 inhibitor, $\mathrm{n}(\%)$ & $132(38)$ \\
\hline Insulin, $\mathrm{n}(\%)$ & $28(8)$ \\
\hline Statin, n $(\%)$ & $181(52)$ \\
\hline RAS inhibitor, $\mathrm{n}(\%)$ & $161(46)$ \\
\hline Eicosapentaenoic acid, n (\%) & $14(4)$ \\
\hline
\end{tabular}

Continuous values are mean $\pm \mathrm{SD}$. HbA1c: hemoglobin A1c; HOMAIR: homeostasis assessment insulin resistance; LDL: low-density lipoprotein; HDL: high-density lipoprotein; eGFR: estimated glomerular filtration rate; d-ROMs: derivatives of reactive oxygen metabolites; $\mathrm{PI}$ : pulsatility index; CCA: common carotid artery; IMT: intima-media thickness; WBPT: whole blood passage time; DPP: dipeptidyl peptidase; RAS: renin-angiotensin system.

forearm using a commercial instrument (AGE Reader ${ }^{\mathrm{TM}}$; DiagnOptics, Groningen, The Netherlands), as previously described [14]. Blood cell counts, plasma glucose concentration, hemoglobin A1c (HbA1c) levels, plasma insulin concentration, serum lipid concentration, estimated glomerular filtration rate (eGFR), and derivatives of reactive oxygen metabolites (d-ROMs) were measured. Blood samples were collected from the antecubital veins in the morning after $12 \mathrm{~h}$ of fasting. Glucose and insulin concentrations were measured using 

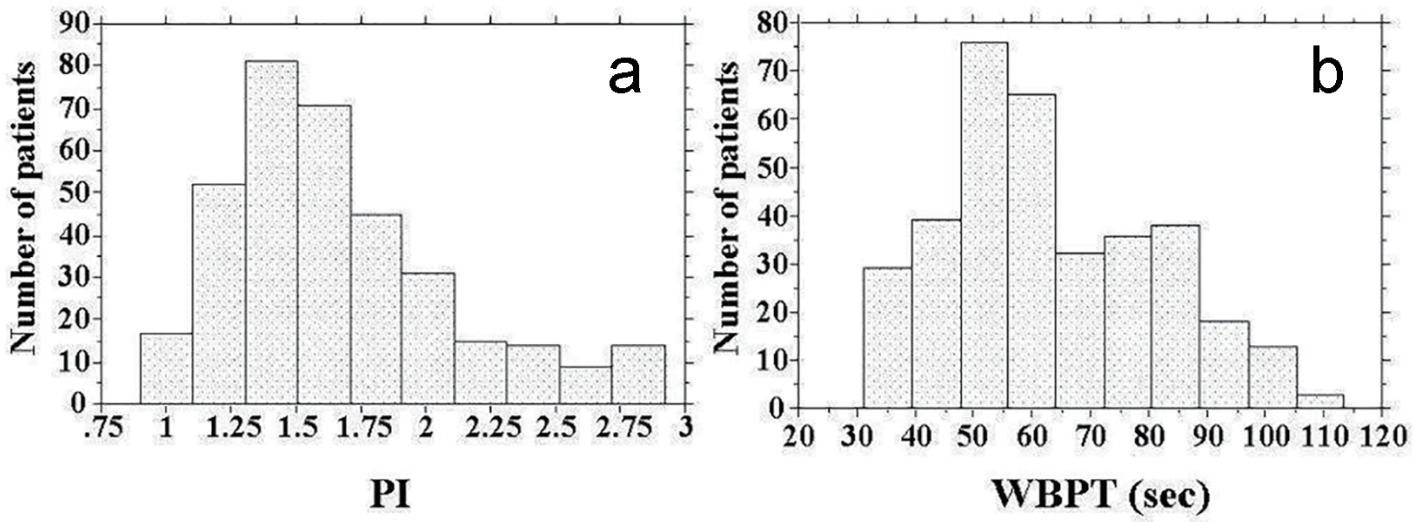

Figure 1. Histogram of PI and WBPT. (a) The mean and median values of PI were $1.69( \pm 0.63$; range, $0.69-2.92)$ and 1.61 , respectively. (b) The mean and median values of WBPT were $62.9( \pm 18.0$; range, $31.2-113.50) \mathrm{s}$ and $59.6 \mathrm{~s}$, respectively. PI: pulsatility index; WBPT: whole blood passage time.

the glucose oxidase method and an enzyme immunoassay, respectively. To estimate insulin resistance, the homeostasis model assessment (HOMA-IR) was calculated as follows [15]: HOMA-IR $=$ (fasting glucose concentration $(\mathrm{mg} / \mathrm{dL}) \times$ fasting insulin concentration $(\mu \mathrm{g} / \mathrm{mL})) / 405$. Total cholesterol and triglyceride concentrations were measured using standard enzymatic methods. High- and low-density lipoprotein cholesterol concentrations were measured using selective inhibition and Friedewald formula, respectively [16]. Participants with a serum triglyceride concentration of $\geq 400 \mathrm{mg} / \mathrm{dL}$ were excluded because this method is accurate below this concentration. eGFR was calculated using the adjusted Modification of Diet in Renal Disease Study equation, which was proposed by the working group of the Japanese Chronic Kidney Disease Initiative [17]. The d-ROMs test, which measures hydroperoxide levels, was used to measure the oxidative stress in vivo using a commercial device (Diacron, Grosseto, Italy) [18].

\section{Statistical analysis}

A commercially available statistical software program (StatView-J 5.0; Hulinks Inc., Tokyo, Japan) was used for all statistical analyses. Continuous variables were expressed as mean \pm SD. Simple regression analysis was performed using Spearman rank correlation. Multivariate analysis was performed using multiple regression or multiple logistic regression. A P value of $<0.05$ was considered as statistically significant.

\section{Results}

Baseline clinical characteristics are shown in Table 1, and the histogram of PI and WBPT is shown in Figure 1. The mean and median values of PI were 1.69 ( \pm 0.63 ; range, 0.69 - 2.92) and 1.61, respectively. Further, the mean and median values of WBPT were 62.9 ( \pm 18.0; range, 31.2 - 113.50) and 59.6 $\mathrm{s}$, respectively. These two parameters were almost normally distributed. The correlation between PI and WBPT is shown in Figure 2. A statistically significant positive correlation was ob- served between PI and WBPT. Correlations among PI, WBPT, and various clinical parameters are shown in Table 2. Age, current smoking status, systolic blood pressure, skin AF, eGFR, d-ROMs, and max-IMT were significantly correlated with PI. On the other hand, sex, body mass index, current smoking status, white blood cell count, red blood cell count, hematocrit, fasting blood glucose levels, immunoreactive insulin levels, HOMA-IR, HbAlc, skin AF, serum triglyceride concentration, d-ROMs test, and max-IMT were significantly correlated with WBPT.

A multiple regression analysis of PI as a subordinate factor was performed with explanatory variables that were significant in univariate analysis. Age, WBPT, max-IMT, current smoking status, d-ROMs test, and skin AF were selected as independent variables for PI (Table 3). To clarify the threshold of WBPT for detecting a high PI, participants were divided into three groups based on WBPT, and multiple logistic regression analysis was performed (Fig. 3). A high PI of CCA was defined as a PI of $\geq$ 2. Patients with high WBPT ( $\geq 70 \mathrm{~s}$ ) had significantly higher

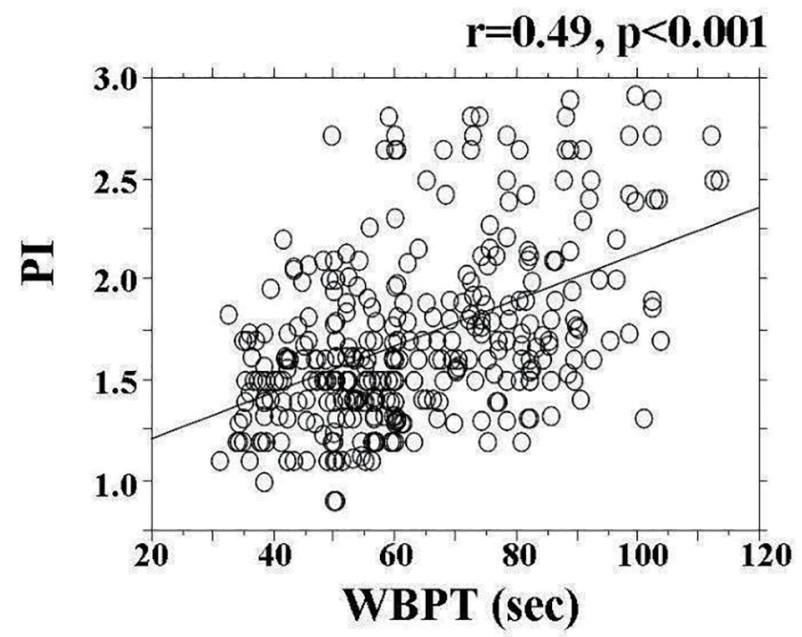

Figure 2. The correlation between PI and WBPT. A statistically significant positive correlation $(r=0.49, P<0.001)$ was observed between PI and WBPT. PI: pulsatility index; WBPT: whole blood passage time. 
Table 2. Relationship Between PI of CCA, WBPT and Various Clinical Parameters

\begin{tabular}{|c|c|c|}
\hline & \multicolumn{2}{|c|}{$\mathbf{r}$} \\
\hline & PI of CCA & WBPT \\
\hline Sex $($ female $=0$, male $=1)$ & 0.10 & $0.17 * *$ \\
\hline Age & $0.22 *$ & 0.06 \\
\hline Body mass index & 0.09 & $0.12 * * *$ \\
\hline Current smoker $($ no $=0$, yes $=1)$ & $0.36^{*}$ & $0.35^{*}$ \\
\hline Systolic blood pressure & $0.25 *$ & 0.03 \\
\hline Diastolic blood pressure & 0.09 & 0.04 \\
\hline White blood cell & 0.06 & $0.16^{* *}$ \\
\hline Red blood cell & 0.10 & $0.17 * *$ \\
\hline Hematocrit & 0.09 & $0.19 * *$ \\
\hline Platelet & 0.08 & 0.09 \\
\hline Fasting blood glucose & 0.06 & $0.22 *$ \\
\hline Immunoreactive insulin & 0.10 & $0.15 * *$ \\
\hline $\mathrm{HbAlc}$ & 0.10 & $0.18 * *$ \\
\hline HOMA-IR & 0.09 & $0.18^{* *}$ \\
\hline Skin autofluorescence & $0.40 *$ & $0.49 *$ \\
\hline Total cholesterol & -0.08 & -0.08 \\
\hline LDL-cholesterol & -0.04 & -0.04 \\
\hline Triglyceride & 0.10 & $0.11 * * *$ \\
\hline HDL-cholesterol & 0.09 & 0.09 \\
\hline eGFR & $-0.11 * * *$ & -0.08 \\
\hline d-ROMs test & $0.44 *$ & $0.40^{*}$ \\
\hline Max-IMT & $0.43 *$ & $0.26^{*}$ \\
\hline Sulfonylurea $($ no $=0$, yes $=1)$ & 0.03 & 0.04 \\
\hline DPP-4 inhibitor $($ no $=0$, yes $=1)$ & 0.05 & 0.06 \\
\hline Insulin $($ no $=0$, yes $=1)$ & 0.07 & 0.07 \\
\hline Statin $($ no $=0$, yes $=1)$ & -0.09 & -0.08 \\
\hline RAS inhibitor $($ no $=0$, yes $=1)$ & -0.09 & -0.09 \\
\hline Eicosapentaenoic acid $($ no $=0$, yes $=1)$ & -0.04 & -0.03 \\
\hline
\end{tabular}

$r$ expressed correlation coefficient. ${ }^{*} P<0.001,{ }^{* *} P<0.01$, ${ }^{* * *} P<0.05$ HbA1c: hemoglobin A1c; HOMA-IR: homeostasis assessment insulin resistance; LDL: low-density lipoprotein; HDL: high-density lipoprotein; eGFR: estimated glomerular filtration rate; d-ROMs: derivatives of reactive oxygen metabolites; PI: pulsatility index; CCA: common carotid artery; IMT: intima-media thickness; WBPT: whole blood passage time; DPP: dipeptidyl peptidase; RAS: renin-angiotensin system.

risk (odds ratio: 5.2; 95\% confidence interval: $2.4-9.2 ; \mathrm{P}<$ 0.001 ) of being detected with a high PI of CCA than those with low WBPT ( $\leq 52.0 \mathrm{~s})$.

\section{Discussion}

This study aimed to clarify the impact of hemorheology assessed by the microchannel method on the PI of CCA in pa-
Table 3. Multiple Regression Analysis for PI of CCA

\begin{tabular}{llll}
\hline Explanatory factor & $\boldsymbol{\beta}$ & t value & P value \\
\hline Age & 0.27 & 5.2 & $<0.001$ \\
WBPT & 0.24 & 5.1 & $<0.001$ \\
Max-IMT & 0.22 & 4.9 & $<0.001$ \\
Current smoker & 0.19 & 4.2 & $<0.001$ \\
d-ROMs test & 0.17 & 3.6 & $<0.001$ \\
Skin autofluorescence & 0.16 & 3.2 & $<0.01$ \\
HOMA-IR & 0.10 & 1.8 & 0.075 \\
HbAlc & 0.08 & 1.6 & 0.148 \\
Sex & 0.07 & 1.5 & 0.179 \\
Hematocrit & 0.06 & 1.3 & 0.193 \\
eGFR & -0.04 & -0.9 & 0.342 \\
Red blood cell & 0.04 & 0.8 & 0.364 \\
Immunoreactive insulin & 0.04 & 0.8 & 0.379 \\
Systolic blood pressure & 0.04 & 0.7 & 0.412 \\
Body mass index & 0.03 & 0.5 & 0.457 \\
White blood cell & 0.03 & 0.4 & 0.610 \\
Fasting blood glucose & 0.02 & 0.1 & 0.910 \\
Triglyceride & -0.05 & -1.0 & 0.254 \\
\hline
\end{tabular}

$R^{2}=0.43, F=44.3, P<0.001(n=349) . ~ H b A 1 c$ : hemoglobin A1c; HOMA-IR: homeostasis assessment insulin resistance; LDL: low-density lipoprotein; HDL: high-density lipoprotein; eGFR: estimated glomerular filtration rate; d-ROMs: derivatives of reactive oxygen metabolites; $\mathrm{PI}$ : pulsatility index; CCA: common carotid artery; IMT: intima-media thickness; WBPT: whole blood passage time; DPP: dipeptidyl peptidase; RAS: renin-angiotensin system.

tients with type 2 diabetes mellitus in terms of the primary prevention of ischemic stroke. The results of multivariate analysis demonstrated a significant relationship between WBPT as a marker of hemorheology and the PI of CCA. In addition, skin AF, oxidative stress, and current smoking status were also significantly related to WBPT and the PI of CCA. Max-IMT is known to be an important risk factor for cardiovascular events, including ischemic stroke; however, the correlation between max-IMT and WBPT was relatively weak, although the correlation coefficient was statistically significant. Thus, WBPT is more closely associated with the PI of CCA than max-IMT.

PI is considered to reflect vascular resistance that is far distal from the point of examination. Therefore, the pathogenesis of small-sized vessels may affect the PI of the proximal arteries, such as the middle cerebral or carotid artery. Thus, it is conceivable that the PI of CCA may also be a surrogate marker of arteriosclerosis in the cerebral arteries [19, 20]. On the other hand, hemorheology estimated using MC-FAN is an in vitro study that uses artificial blood vessels, and the vessel lumen measures $<10 \mu \mathrm{m}$. Thus, evaluating hemorheology using MC-FAN is assumed to correspond to small-sized vessels, including capillary vessels. Therefore, the significant relationship observed between WBPT and the PI of CCA in this study suggests that the PI of CCA reflects an increase in vascular resistance due to the impairment of hemorheology in the cer- 


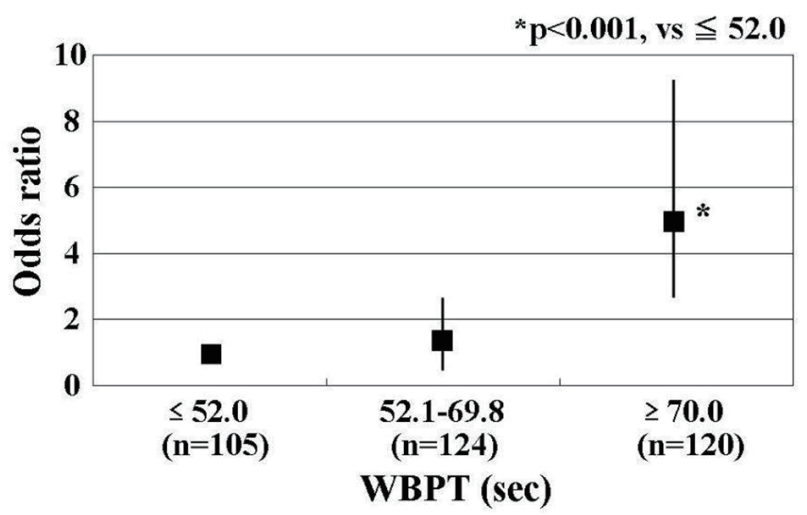

Figure 3. Results of multiple logistic regression analysis for high PI. Patients with high WBPT ( $\geq 70 \mathrm{~s}$ ) had significantly higher risk (odds ratio, 5.2; 95\% confidence interval, $2.4-9.2 ; \mathrm{P}<0.001)$ of being detected with a high PI of CCA than those with low WBPT ( $\leq 52.0 \mathrm{~s})$. A high PI of CCA was defined as a PI of $>2$. Adjustment factors: age, max-IMT, current smoker, d-ROMs test, and skin autofluorescence. PI: pulsatility index; WBPT: whole blood passage time.

ebral small-sized vessels. The relationship between cerebral small-sized vessels and ischemic stroke has been reported in recent years [21]. Thus, high WBPT can be used to predict the incidence of ischemic stroke in patients with type 2 diabetes mellitus, indicating that measures to improve WBPT in early stages should be employed for preventing ischemic stroke.

Among the glucose-related factors, only skin AF was found to be an independent variable of PI. Furthermore, skin AF also exhibited a significant relationship with WBPT in this study. The level of skin pentosidine, which is a major component of AGEs on the volar side of the forearm, has been found to correlate with skin AF by skin biopsy [22]. Small-sized vessels are considered to be present on the skin. Therefore, significant relationship between skin AF and WBPT possibly reflects the accumulation of AGEs in tissues due to the impairment of hemorheology in small-sized vessels. Some basic studies have reported that AGEs influence hemorheology by mechanisms, such as leukocyte-endothelial interaction and platelet aggregation $[23,24]$. Furthermore, the receptors of AGEs (RAGEs) are expressed in the endothelium of brain capillaries and smallsized arteries, and under pathophysiological conditions, such as those associated with the accumulation of RAGE ligands on blood vessels, RAGEs are increased [25]. The impairment of hemorheology in brain small-sized vessels may be caused by an increase in RAGEs. Thus, the results of the present study and previous studies can be interpreted to mean that AGEs and the impairment of hemorheology are associated with each other not only on the volar side of the forearm but also in the neurovascular system, consequently leading to neurovascular damage and a risk of ischemic stroke. Genevieve et al examined the relationship between skin $\mathrm{AF}$ and $\mathrm{HbA} 1 \mathrm{c}$ levels [26]. They measured HbAlc levels every 6 months and reported that skin AF levels are significantly related to the means of the last 5 and $10 \mathrm{HbA} 1 \mathrm{c}$ values. In addition, Lee et al reported that the PI of the internal carotid and middle cerebral arteries using Doppler ultrasonography are significantly related to the duration of diabetes. Thus, the results of the present study and previous study indicate that we should maintain long-term glucose control to improve the hemorheology or PI of cerebral and carotid arteries in patients with type 2 diabetes mellitus.

Some studies have reported that oxidative stress plays an important role in neurovascular dysfunction [27-29]. In the present study, d-ROMs test as a marker of oxidative stress in vivo exhibited significant correlation with the PI of CCA by multivariate analysis. Furthermore, d-ROMs test exhibited a significant relationship with WBPT. Oxidative stress causes the impairment of hemorheology through several mechanisms, such as platelet aggregation and increase in plasma viscosity $[30,31]$. In contrast, renin-angiotensin system inhibitors and statins decrease oxidative stress in vivo $[32,33]$. Furthermore, large prospective studies have also reported the clinical usefulness of these drugs in preventing ischemic stroke [34, 35]. The results of this cross-sectional study indicate that there is no significant relationship between medications and PI or WBPT. However, interventional studies are warranted to examine the effect of medications on d-ROMs test, PI, and WBPT. Consequently, we may discover new applications of those drugs.

It is well known that smoking affects hemorheology. Furthermore, some clinical studies have demonstrated a relationship between smoking and WBPT $[12,36]$. Alzimami et al have reported a significant positive correlation between daily cigarette smoking and PSV and a negative correlation between smoking and EDV, as assessed using CCA ultrasound scanning, suggesting a relationship between the high PI of CCA and smoking [37]. The results of the present study also demonstrated that current smoking was an independent determination factor for the PI of CCA and that current smoking exhibited a significant relationship with WBPT. Smoking is also known to increase oxidative stress and increase AGEs [38, 39]. Thus, smoking cessation is strongly recommended for the primary prevention of ischemic stroke in patients with type 2 diabetes mellitus.

It is useful to have a target value for predicting the incidence of ischemic stroke in clinics. In one study, to clarify the clinical usefulness of measuring WBPT, participants were divided into three groups based on WBPT and multiple logistic regression analysis was performed for detecting a high PI $(\geq$ 2) of CCA, which has been reported to be an independent risk factor for ischemic stroke [5]. The results of the present study indicated that patients with WBPT of $\geq 70.0 \mathrm{~s}$ exhibited significantly higher risk of having a high PI of CCA than those with WBPT of $\leq 52.0 \mathrm{~s}$. Although this was a cross-sectional study, we believe that it is possible to evaluate the risk of ischemic stroke by measuring WBPT. Further, we suggest that ischemic stroke can be prevented by interventions, such as medication or lifestyle modification in patients with high WBPT of $\geq 70.0 \mathrm{~s}$.

\section{Limitations}

This study has several limitations. First, the drug treatments for diabetes mellitus, hypertension, and/or dyslipidemia may have influenced the study results. Second, this was a singlecenter cross-sectional study with a relatively small population. A prospective study involving a larger number of patients is necessary to confirm the effectiveness of interventions, such 
as antioxidant administration or smoking cessation, in improving WBPT or the PI of CCA. Third, hemorheology estimated using MC-FAN is an in vitro study that uses artificial blood vessels; therefore, the obtained hemorheological data were different from those obtained in vivo because of the influence of vascular factors, such as endothelial cells or smooth muscle cells. However, the results of the present study indicate that WBPT, which is estimated using MC-FAN, is a useful biomarker to evaluate neurovascular resistance in clinic. Finally, an extensive examination of clinical studies will be required in the future to investigate the significance of WBPT as a risk factor for cardiovascular events, including ischemic stroke.

\section{Conclusions}

In conclusion, the present study indicated that WBPT estimated by the microchannel method is an important determination factor for the PI of CCA, suggesting that the increase in WBPT can potentially predict the incidence of ischemic stroke in patients with type 2 diabetes mellitus.

\section{Competing Interests}

Author has no competing interests.

\section{Grant Support}

None.

\section{Financial Disclosure}

None.

\section{References}

1. Kannel WB, McGee DL. Diabetes and cardiovascular disease. The Framingham study. JAMA. 1979;241(19):20352038.

2. Arboix A, Rivas A, Garcia-Eroles L, de Marcos L, Massons J, Oliveres M. Cerebral infarction in diabetes: clinical pattern, stroke subtypes, and predictors of in-hospital mortality. BMC Neurol. 2005;5(1):9.

3. Weih M, Amberger N, Wegener S, Dirnagl U, Reuter T, Einhaupl K. Sulfonylurea drugs do not influence initial stroke severity and in-hospital outcome in stroke patients with diabetes. Stroke. 2001;32(9):2029-2032.

4. Lee KY, Sohn YH, Baik JS, Kim GW, Kim JS. Arterial pulsatility as an index of cerebral microangiopathy in diabetes. Stroke. 2000;31(5):1111-1115.

5. Nakatou T, Nakata K, Nakamura A, Itoshima T. Carotid haemodynamic parameters as risk factors for cerebral infarction in Type 2 diabetic patients. Diabet Med. 2004;21(3):223-229.
6. Tzoulaki I, Murray GD, Lee AJ, Rumley A, Lowe GD, Fowkes FG. Relative value of inflammatory, hemostatic, and rheological factors for incident myocardial infarction and stroke: the Edinburgh Artery Study. Circulation. 2007;115(16):2119-2127.

7. Matsuo K, Ueda Y, Nishio M, Hirata A, Asai M, Nemoto $\mathrm{T}$, Kashiwase K, et al. Thrombogenic potential of whole blood is higher in patients with acute coronary syndrome than in patients with stable coronary diseases. Thromb Res. 2011;128(3):268-273.

8. Kikuchi Y, Sato K, Mizuguchi Y. Modified cell-flow microchannels in a single-crystal silicon substrate and flow behavior of blood cells. Microvasc Res. 1994;47(1):126139.

9. Kamada H, Okamoto T, Hayashi T, Suzuki K. An in vitro method for screening anti-platelet agents using a microchannel array flow analyzer. Biorheology. 2010;47(2):153-161.

10. Satoh N, Kotani K, Wada H, Himeno A, Shimada S, Sasaki Y, Yamada K, et al. Unfavorable blood rheology is closely associated with arterial stiffness in obese patients. Endocr J. 2009;56(7):915-918.

11. Yagi H, Sumino H, Aoki T, Tsunekawa K, Araki O, Kimura T, Nara M, et al. Impaired blood rheology is associated with endothelial dysfunction in patients with coronary risk factors. Clin Hemorheol Microcirc. 2016;62(2):139150.

12. Hitsumoto T. Factors affecting impairment of blood rheology in obese subjects. J Cardiol. 2012;60(5):401-406.

13. Yoshida S, Aihara K, Azuma H, Uemoto R, SumitomoUeda Y, Yagi S, Ikeda Y, et al. Dehydroepiandrosterone sulfate is inversely associated with sex-dependent diverse carotid atherosclerosis regardless of endothelial function. Atherosclerosis. 2010;212(1):310-315.

14. Meerwaldt R, Hartog JW, Graaff R, Huisman RJ, Links TP, den Hollander NC, Thorpe SR, et al. Skin autofluorescence, a measure of cumulative metabolic stress and advanced glycation end products, predicts mortality in hemodialysis patients. J Am Soc Nephrol. 2005;16(12):3687-3693.

15. Matthews DR, Hosker JP, Rudenski AS, Naylor BA, Treacher DF, Turner RC. Homeostasis model assessment: insulin resistance and beta-cell function from fasting plasma glucose and insulin concentrations in man. Diabetologia. 1985;28(7):412-419.

16. Friedewald WT, Levy RI, Fredrickson DS. Estimation of the concentration of low-density lipoprotein cholesterol in plasma, without use of the preparative ultracentrifuge. Clin Chem. 1972;18(6):499-502.

17. Imai E, Horio M, Nitta K, Yamagata K, Iseki K, Hara $\mathrm{S}$, Ura $\mathrm{N}$, et al. Estimation of glomerular filtration rate by the MDRD study equation modified for Japanese patients with chronic kidney disease. Clin Exp Nephrol. 2007;11(1):41-50.

18. Cesarone MR, Belcaro G, Carratelli M, Cornelli U, De Sanctis MT, Incandela L, Barsotti A, et al. A simple test to monitor oxidative stress. Int Angiol. 1999;18(2):127-130.

19. Fukuhara T, Hida K. Pulsatility index at the cervical internal carotid artery as a parameter of microangiopa- 
thy in patients with type 2 diabetes. J Ultrasound Med. 2006;25(5):599-605.

20. Lee KO, Lee KY, Lee SY, Ahn CW, Park JS. Lacunar infarction in type 2 diabetes is associated with an elevated intracranial arterial pulsatility index. Yonsei Med J. 2007;48(5):802-806.

21. Pantoni L. Cerebral small vessel disease: from pathogenesis and clinical characteristics to therapeutic challenges. Lancet Neurol. 2010;9(7):689-701.

22. Meerwaldt R, Graaff R, Oomen PH, Links TP, Jager JJ, Alderson NL, Thorpe SR, et al. Simple non-invasive assessment of advanced glycation endproduct accumulation. Diabetologia. 2004;47(7):1324-1330.

23. Morigi M, Angioletti S, Imberti B, Donadelli R, Micheletti G, Figliuzzi M, Remuzzi A, et al. Leukocyte-endothelial interaction is augmented by high glucose concentrations and hyperglycemia in a NF-kB-dependent fashion. J Clin Invest. 1998;101(9):1905-1915.

24. Hasegawa Y, Suehiro A, Higasa S, Namba M, Kakishita E. Enhancing effect of advanced glycation end products on serotonin-induced platelet aggregation in patients with diabetes mellitus. Thromb Res. 2002;107(6):319-323.

25. Zlokovic BV. New therapeutic targets in the neurovascular pathway in Alzheimer's disease. Neurotherapeutics. 2008;5(3):409-414.

26. Genevieve M, Vivot A, Gonzalez C, Raffaitin C, Barberger-Gateau P, Gin H, Rigalleau V. Skin autofluorescence is associated with past glycaemic control and complications in type 1 diabetes mellitus. Diabetes Metab. 2013;39(4):349-354.

27. Kontos HA. Oxygen radicals in cerebral ischemia: the 2001 Willis lecture. Stroke. 2001;32(11):2712-2716.

28. Kinouchi H, Epstein CJ, Mizui T, Carlson E, Chen SF, Chan PH. Attenuation of focal cerebral ischemic injury in transgenic mice overexpressing $\mathrm{CuZn}$ superoxide dismutase. Proc Natl Acad Sci U S A. 1991;88(24):1115811162.

29. Cherubini A, Ruggiero C, Polidori MC, Mecocci P. Potential markers of oxidative stress in stroke. Free Radic Biol Med. 2005;39(7):841-852.

30. Tzoulaki I, Murray GD, Lee AJ, Rumley A, Lowe GD, Fowkes FG. Inflammatory, haemostatic, and rheological markers for incident peripheral arterial disease: Edinburgh Artery Study. Eur Heart J. 2007;28(3):354-362.

31. Podrez EA, Byzova TV, Febbraio M, Salomon RG, Ma Y, Valiyaveettil M, Poliakov E, et al. Platelet CD36 links hyperlipidemia, oxidant stress and a prothrombotic phenotype. Nat Med. 2007;13(9):1086-1095.

32. Lodovici M, Bigagli E, Tarantini F, Di Serio C, Raimondi L. Losartan reduces oxidative damage to renal DNA and conserves plasma antioxidant capacity in diabetic rats. Exp Biol Med (Maywood). 2015;240(11):1500-1504.

33. Hitsumoto T, Iizuka T, Takahashi M, Nakamura K, Shimizu K, Satoh S, Sugiyama Y, et al. [A relationship between insulin resistance and reduction in oxidative stress in vivo by atorvastatin]. J Cardiol. 2004;44(6):233-242.

34. Dahlof B, Devereux RB, Kjeldsen SE, Julius S, Beevers G, de Faire U, Fyhrquist F, et al. Cardiovascular morbidity and mortality in the Losartan Intervention For Endpoint reduction in hypertension study (LIFE): a randomised trial against atenolol. Lancet. 2002;359(9311):995-1003.

35. Pedersen TR, Kjekshus J, Berg K, Haghfelt T, Faergeman $\mathrm{O}$, Faergeman G, Pyorala K, et al. Randomised trial of cholesterol lowering in 4444 patients with coronary heart disease: the Scandinavian Simvastatin Survival Study (4S). 1994. Atheroscler Suppl. 2004;5(3):81-87.

36. Shimada S, Hasegawa K, Wada H, Terashima S, SatohAsahara N, Yamakage H, Kitaoka S, et al. High blood viscosity is closely associated with cigarette smoking and markedly reduced by smoking cessation. Circ J. 2011;75(1):185-189.

37. Alzimami KS, Mahmoud MZ, Sulieman A. Evaluation of common carotid artery changes in Saudi current smokers using medical ultrasound (B-mode and Doppler). J Med Ultrason (2001). 2014;41(3):351-357.

38. Csiszar A, Podlutsky A, Wolin MS, Losonczy G, Pacher $\mathrm{P}$, Ungvari Z. Oxidative stress and accelerated vascular aging: implications for cigarette smoking. Front Biosci (Landmark Ed). 2009;14:3128-3144.

39. Yamagishi S, Matsui T, Nakamura K. Possible involvement of tobacco-derived advanced glycation end products (AGEs) in an increased risk for developing cancers and cardiovascular disease in former smokers. Med Hypotheses. 2008;71(2):259-261. 